\title{
Threat in a Two-Person Game ${ }^{1}$
}

\author{
Melvin Guyer and Anatol Rapoport \\ The University of Michigan
}

\begin{abstract}
A two-person nonzero-sum game which provides one player with a threat option is experimentally investigated in this study. In the game, both players have a dominating strategy choice but the "natural" outcome of the game, defined as the intersection of dominating strategy choices, gives one player his largest payoff and the other player his next to smallest. However, the "dissatisfied" player (the one who does not receive his largest payoff at the natural outcome) can, by switching his strategy choice, reduce the other's payoffs but only at a cost to himself. The dissatisfied player's ability to lower the other's payoffs constitutes a "threat."

It was found that in repeated trials of play of this game, those players who were likely to carry out their threats were those who won the most concessions from the other. The results of this study suggest that a threat-appeasement, punishment-capitulation interaction develops between the players. That is, the existence of the threat option for one player leads the other to make concessions in order to avoid punishment and, once punishments are carried out, they too are likely to result in concessions for the punishers.

It is also noted that the "sure-thing" principle fails to provide an adequate description of the strategy choice behavior of naive subjects.
\end{abstract}

Two-person nonzero-sum games have been used in a variety of experimental situations (for reviews of the literature see Gallo \& McClintock, 1965; Rapoport \& Orwant, 1962) to study mixed-motive behavior in social interaction. The major concern of most of these studies has been with the motives of cooperation and competition (as in the Prisoner's Dilemma game) and brinkmanship and pre-emption (as in the game of Chicken). These games, however, represent only two among a large number of interesting mixed-motive social interaction situations which can be represented by means of two-person nonzero-sum games.

A. recent publication (Rapoport \& Guyer, 1966), presented a taxon-

${ }^{1}$ The research work on which this paper is based was supported by National Institutes of Health, Grant NIH-MH 12880-02 (1967-68), "Psychology of Conflict. Involving Mixed-Motive Decisions," and NIH-MH 15942-01, "Psychology of Confict and Cooperation." 
omy of all two-person two-strategy games in which each player had a strong preference-ordering over outcomes. It was shown that there are exactly 78 such strategically different games. Many of these games upon examination turned out to capture interesting aspects of mixed-motive behavior; yet, to the knowledge of the present authors, few of these games have been the subject of experimental investigation. It is the purpose of this paper to report some empirical findings concerning one such game. This game is a member of the category of games having a single threat-vulnerable equilibrium (see "Taxonomy" cited above). In such games, one player can induce the other to shift his strategy if the other can be made to see that it is to his advantage to shift rather than to suffer the consequences of the other's shifting. This type of inducement functions as a threat, since one player has the potential of punishing the other if that other does not make a concession to the first player. The nature of threat in these games shares characteristics of real-world threats in that, once the threat is carried out, the motivation to comply, or to make concessions by the other, is reduced or eliminated. This is typical of realworld threats such as the demand for ransom to forestall the killing of a kidnapped victim, or the demand for blackmail under the threat of disclosure of damaging information, etc. In both cases, the threats lose their efficacy if they are carried out. That is, one does not pay ransom to a kidnapper if the victim has been killed nor does one make payoffs to a blackmailer after damaging information has been made public.

This paper reports the results of an experiment in which ten pairs of naive subjects each played 300 repeated trials of a threat-vulnerable game.

\section{METHOD}

\section{Subjects}

Subjects were 20 undergraduate males at the University of Michigan recruited from a voluntary paid subject pool. In this experiment each subject was paid $\$ 1.35$ for the one-hour experimental session. In addition to their hourly earnings, subjects were informed that in the experiment they could either win or lose money, depending on how they played, and that these gains or losses would be added to or subtracted from their hourly wage.

\section{Procedure}

Subjects were randomly assigned to pairs with the provision that members of each pair were unacquainted with one another.

At the start of an experimental session the pair of subjects enter a cubicle where they are seated, separated from one another by a solid partition. Each subject is provided with a response box which includes a display of the payoff matrix and response buttons which the subjects push to indicate their strategy choices on each trial.

The subjects were instructed as to how to read the payoff matrix and how to indicate their responses on each trial. The instructions also informed the players that 
the points which they won or lost would be converted into cash at the rate of $1 / 50$. per point at the end of the experiment. The instructions were intended to give the subjects an individualistic orientation toward the task; in essence, each was told to make as many points as possible for himself without regard to the number of points earned by the other.

The experimenter read instructions to the subjects via an intercom system and monitored the experimental session from a separate room. After the subjects received instructions they played repeated trials of the game. When both players had responded on a trial, the outcome defined by their strategy choices was made known to them by having the appropriate cell of the payoff matrix on each p'ayer's response box light up. Thus, aftcr cach trial of play each subject was given feedback conceraing his own and the other's payoffs. The recording of responses and fecdback to the players after each trial was computer controlled. After each b.ock of 25 trials, the experimenter reported to each subject the sum of his payoffs for that block of trials.

Upon completion of 300 trials of play the subjects were given their net payoffs. These were determined by taking the points each had accumulated and converting them to cash, which was then added to or subtracted from the hourly wage of $\$ 1.35$.

\section{The Game Matrix}

As defined, Game 21 is a threat-vulnerable game in which each player has a dominating strategy choice. Since a dominating strategy choice is one which guarantecs to the player a larger payoff than any of his other strategies, regardless of what the other player does, its selection is prescribed by normative decision-making criteria (Luce \& Raiffa, 1957, p. 96).

In the "Taxonomy," games are defined only in terms of the players' preferenceordering over outcomes. Thus, there can be as many versions of a game as there are assignments of monetary payoffs which are consistent with the players' preferenceorderings in that game. The version of Game 21 played by the subjects in this study is shown in Matrix 1, where the first entry in a cell is Row's payoff and the second is Column's.

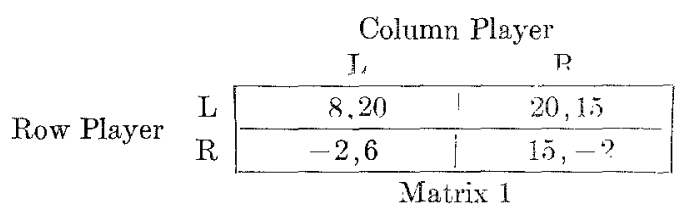

Here, the L strategy choice dominates the $\mathrm{R}$ strategy choice for both Row and Column players. Thus rational decision-making considerations dictate that, on a single play of the game, each player should select his L strategy; this results in the ontcome LL which, as can be seen from Matrix 1, gives Row player 8 points and Column player 20 points. Unfortunately for Row player, this outcome, dictated by rationality, gives him his next to smallest payoff while Column receives his largest payoff. This is more readily apparent if the payoffs in Matrix 1 are replaced by their ordinal values, each player's preference-ordering over outcomes being $4>3>2>1$. This representation of Game 21 is shown in Matrix 2.

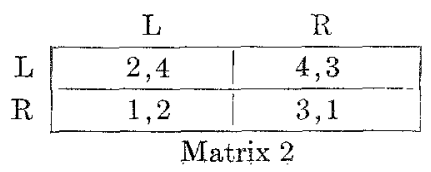


Looking at Matrix 2, it is evident that on a single play of this game, without preplay communication, Column ought to make his L strategy choice and Row ought to choose L (we are speaking now of "rational players"). In fact, since both players have dominating strategy choices, neither player would alter his choice from $\mathrm{L}$ to $\mathrm{R}$ even if he knew beforehand the other player's strategy choice, be it $L$ or $R$. So, in a single play of Game 21, Row must be content with his next-to-smallest payoff while Column receives his largest payoff. The game is "unfair" to Row but nothing can be done about it.

In repeated plays of Game 21, however, the strategic characteristics become different: the LI outcome now becomes threat-vulnerable; that is, Row may shift from $\mathrm{L}$ to $\mathrm{R}$. Such a shift would takc the outcome to $\mathrm{RL}$ which would reduce Column's payoff from his most preferred to his next-to-least preferred outcome. It is the possibility of Row making such a shift that constitutes Row's threat to Column. The purpose of Row's threat would be to induce Column to switch from $L$ to $R$, taking the outcome to LR where Column receives his next-to-largest payott while giving Row his largest payoff. Column may perceive the threat implicit in the game and, in the face of the possibility of Row's shift from $L$ to $R$, may either refuse to shift from $L$ to $R$, thereby suffering the consequences if Row carries out his threat, or may shift from $\mathrm{L}$ to $\mathrm{R}$ in order to deter Row from carrying out his threat. If Column does not shift from $L$ to $R$, and if Row carries out his threat, Column may either subsequently give in to Row's demand for his largest payoff and switch to $R$, or may refuse to accede to Row's threat and persist in $\mathbf{L}$, his dominating strategy choice.

Correspondingly, Row may choose not to carry out his threat against Column, since his use of threat takes him to his smallest payoff; or he may repeatedly carry out his threat against Column until Column accedes; or, since carrying out his threats against Column may be unsuccessful in gaining concessions, Row may revert to his L. strategy choice.

Another possible type of strategy choice pattern available to the players is one in which Row always selects his dominating (L) strategy choice while Column periodically shifts back and forth between his $L$ and $R$ strategy choices. Such a pattern would yield relatively high payoffs to both players and Column could, by playing appropriate proportions of each of his responses, produce an approximately equal distribution of payoffs to himself and the other. Though this type of pattern seems fair and equitable, apparently none of the pairs of players in this study chose to play this way. We can only conjecture that Column finds it difficult to unilaterally depart from his dominating strategy choice when not faced with a Row player who threatens. For, in the short run at lcast, Column gains whenever he yiclds to the tcmptation to continue in $\mathrm{L}$ rather than making a shift to $\mathrm{R}$ as would be called for by an "equitable" pattern of play.

In examining the strategy choice behavior of ten pairs of subjects each playing 300 repeated trials of Matrix 1 , we are concerned primarily with two questions about the strategy choice behavior of the players:

1. Does Row player use his threat option against Column in an effort to gain his (Row's) largest payoff?

2. Is Row's use of threat successful in obtaining concessions from Column?

In seeking the answers, certain restatements and reformulations of these questions will be required; however, it must be remembered that these two questions focus on the fundamental strategic characteristics of the threat-appeasement conflict which is captured in Game 21. 


\section{RESULTS}

Table 1 shows the mean percentages of each of the four possible outcomes in Game 21 for ten pairs of subjects. These percentages are based on the entire 300 trials of play.

From this table it can be seen that Row player does in fact carry out his "threat" against Column. The average percentage of plays on which Row departed from his dominating strategy choice is $13 \%$. Also, Column player does depart from his dominating strategy choice so as to give Row his largest payoff. The percentage of outcomes in which Column does this is $28 \%$. The fact that the LL outcome (which is dictated by normative decision-making criteria) occurred only $62 \%$ of the time indicates the degrce to which such rational dccision-making considcrations fail to account for the behavior of actual players in mixed-motive games.

A question immediately raised by the results in Table $I$ is whether (and to what extent) Column gives Row his largest outcome to prevent Row from carrying out his threat, or whether Column gives Row his largest payoff as a consequence of Row's carrying out his threat. For discussion it will be convenient to refer to Colymn's avoiding Row's threat as "appeasement" and Column's giving Row his largest payoff immediately after Row's threats are carried out as "capitulation." With this terminology the question can be restated as "When LR outcomes occur, to what extent do they represent appeasement and to what extent capitulation?"

One approach to this question is in terms of the way in which Column responds to Row's carrying out of his threat. If LR outcomes (those in which Row receives his largest payoff) come about from capitulation by Column, there should be a positive correlation, taken across pairs of players, between RL and LR outcomes. (In computing this correlation. we ignore the two pairs in which Row never carried out his threat against Column.) The Pearson product-moment correlation between the frequency of LR and RL outcomes (for 300 trials of play) across the remaining eight pairs of players is $r=.83$ (significant beyond $p=.05$ ). A reasonable interpretation of this rather high correlation is that the carrying out of threats by Row results in capitulations by Column. It should be noted that this correlation implies that the use of threats by Row is effective in obtaining concessions from Column even though these

TABLE 1

Mean Percentages of the Four Outcomes of Game 21 for 300 Trials

\begin{tabular}{cccc}
\hline TI & $\mathrm{LR}$ & $\mathrm{RL}$ & $\mathrm{RR}$ \\
\hline 62 & 24 & 09 & 04 \\
\hline
\end{tabular}


concessions require that Column depart from his dominating strategy choice.

The question as to whether LR outcomes result from appeasement or from capitulation is not answered simply in terms of the correlation between LR and RL outcomes. Other aspects of the data indicate that appeasement also plays a role. As sean from Table 1, in the combined data of ten pairs of players, LR outcomes occurred more than twice as frequently as RL outcomes. The excess of $L R$ over RL outcomes indicates that appeasement is a factor in Column's concessions to Row because it shows that Column gave Row his largest payoff to an extent that exceeded Row's carrying out of threats. Such additional concessions serve only to appease Row, that is, to deter him from carrying out future threats.

Figure 1 shows the percentage of LR and RL outcomes for each pair of players, from which can be seen that the typical pattern for a pair has the LR outcomes exceeding the RL outcomes. Four pairs are exceptions, two of which are especially interesting. Note that in Pair 3, all 300 outcomes were LR; that is, Column appeased Row on every trial of the game; consequently Row had no need ever to carry out his threat. In Pair 4, Row never carried out his threat and Column never appeased: all outcomes for this pair were LL; that is, each player behaved "rationally" by never departing from his dominating strategy choice.

Another way of looking at the strategic interaction taking place between Row and Column is in terms of the conditional probability of either player selecting his dominating (or non-dominating) strategy choice given each of the four possible outcomes that could occur on the previous

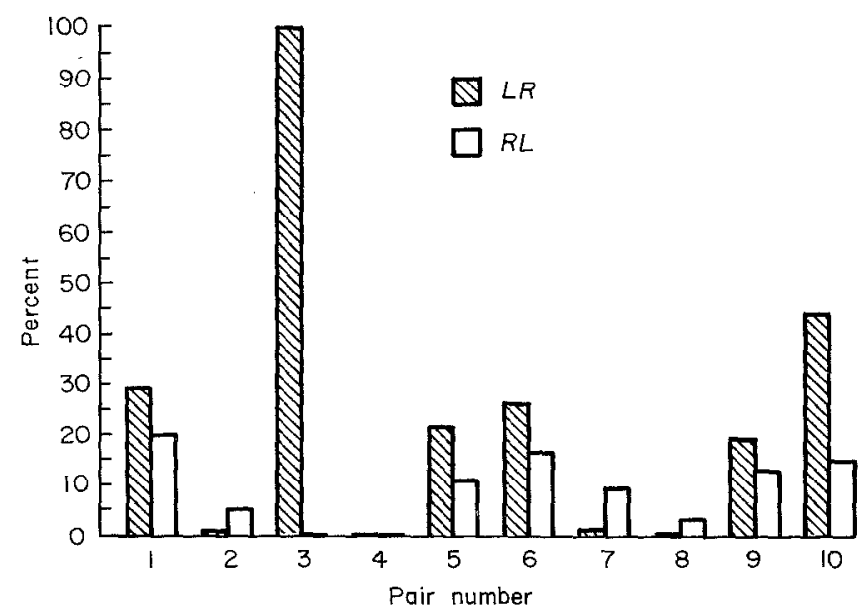

FIG. 1. Percentages of $\mathrm{LR}$ and RL outcomes for each of the ten pairs of players for the 300 repeated trials of play. 
trial. These various conditional probabilities can be interpreted as propensities to engage in certain strategic maneuvers in the game. In order to clarify the discussion of these propensities, the following example of one of them is given:

$p\left(\mathrm{R}_{1} \mid \mathrm{L}_{1} \mathrm{~L}_{2}\right)$ defines the conditional probability of player 1 (Row) selecting his $\mathrm{R}$ strategy choice on trial $n$, given that both he and player 2 (Column) selected their $\mathrm{L}$ strategy choices on the preceding trial, $n-1$. Since there are four possible outcomes on trial $n-1$, and two players, there is a total of eight such independent conditional probabilities or propensities. Some of these propensities may be given a strategic interpretation. In the example above, $p\left(\mathrm{R}_{1} \mid \mathrm{L}_{1} \mathrm{~L}_{2}\right)$ represents the propensity that Row will carry out a threat against Column. Correspondingly, $p\left(R_{2} \mid L_{1} L_{2}\right)$ represents the propensity that Column will appease Row so as to avoid suffering the consequences of Row's threat. (Other conditional probabilities will be introduced and given interpretation as strategic propensities as the discussion demands.)

By combining the data of all Row players, a mean value of $p\left(R_{1} \mid L_{1} L_{2}\right)$ is obtained. Similarly, a mean value of $p\left(R_{2} \mid L_{1} L_{2}\right)$ is found for the combined data from the ten Column players. On the basis of ten Row players and ten Column players, each playing 300 repeated trials of Game 21, the mean propensity for Row carrying out his threat against Column. $p\left(R_{1} \mid L_{1} L_{2}\right)$, is .10. The mean propensity that Column will appease Row, $p\left(R_{2}, L_{1} L_{2}\right)$, is .12. These two figures confirm what was stated previously: Row does sometimes carry out his threat against Column, and Column does make concessions to Row as appeasement. The point is also underscored here that both Row and Column players do depart, to a considerable extent, from their rational or dominating strategy choices.

Table 2 shows the complete matrix of intercorrelations between the Row and Column players' propensities to select their non-dominating strategy choices on trial $n$ given each of the four possible outcomes that could occur on trial $n-I$.

Here we examine more closely the relationship between $p\left(\mathrm{R}_{1} \mid \mathrm{L}_{1} \mathrm{~L}_{2}\right)$ and $p\left(R_{2} \mid \mathrm{L}_{1} \mathrm{~L}_{1}\right)$. The product-moment correlation between these propensities for Row and Column can be determined across the pairs of players. Taken across the nine pairs of players for whom these propensities are defined, we find that $r=.69$ (significant beyond $p=.05$ level). This can be interpreted as confirming that Row's likelihood of using threat is positively related to Column's granting of concessions. And, as stated earlier, concessions so obtained represent capitulation by Column.

Column's likelihood of capitulating to Row is more directly captured by $p\left(\mathbf{R}_{2}, \mathbf{R}_{1} L_{2}\right)$. In words, this is the probability that Column will select his non-dominating strategy choice following a trial on which Row car- 
TABLE 2

Matrix of Intercorrelations Between Row's (Player 1) and Column's (Player 2) Propenstties to Select Their Non-dominating Strategy Chorce on Trial $n$ Given Each of the Four Possible Outcomes

That Could Occur on Trial $n-1$

(Correlations are taken across pairs of players and the propensities for each player are based on 300 trials of play.)

\begin{tabular}{lcccc} 
& $p\left(\mathrm{R}_{2} \mid \mathrm{L}_{1} \mathrm{~L}_{2}\right)$ & $p\left(\mathrm{R}_{2} \mid \mathrm{R}_{1} \mathrm{R}_{2}\right)$ & $p\left(\mathrm{R}_{2} \mid \mathrm{R}_{1} \mathrm{~L}_{2}\right)$ & $p\left(\mathrm{R}_{2} \mid \mathrm{R}_{1} \mathrm{R}_{2}\right)$ \\
\hline$p\left(\mathrm{R}_{1} \mid \mathrm{I}_{1} \mathrm{I}_{1_{2}}\right)$ & $.69^{*}$ & .60 & $.96^{* *}$ & $.92^{* *}$ \\
$p\left(\mathrm{R}_{1} \mid \mathrm{L}_{1} \mathrm{R}_{2}\right)$ & -.38 & .01 & -.46 & -.48 \\
$p\left(\mathrm{R}_{1} \mid \mathrm{R}_{1} \mathrm{~L}_{2}\right)$ & .29 & -.31 & .32 & -.03 \\
$p\left(\mathrm{R}_{1} \mid \mathrm{R}_{1} \mathrm{R}_{2}\right)$ & -.41 & -.44 & -.62 & -.61 \\
\hline- & - &
\end{tabular}

$* p<.05$.

$* * p<.01$.

ried out his threat. In fact, this probability can be taken as Column's propensity to capitulate to Row. The combined data of Column players yields a mean value of .23 for this propensity. Thus, though Column both appeases and capitulates, his probability of capitulating is practically twice as great as his probability of appeasing.

The method of correlating Row and Column's strategic propensities can be used to shed light on other aspects of the players' interaction in Game 21. One such aspect is the relationship between Row's readiness to carry out his threats, and Column's readiness to capitulate once such threats have been carried out. These two propensitics arc $p\left(R_{1} \mid L_{1} L_{2}\right)$ and $p\left(R_{2} \mid R_{1} L_{2}\right)$, respectively, for Row and Column. Now, it should be pointed out that the question here is not whether Column capitulates more often when Row threatens more often; rather it is a question about conditional events. Thus the question becomes: What is the relationship between Row's likelihood of carrying out his threat and Column's likelihood of capitulating, given a threat has been carried out? $\mathrm{Or}$, to put it another way, does Column more readily capitulate when faced with a Row player who readily carries out his threat?

To answer these questions we determine the product-moment correlation between $p\left(\mathrm{R}_{1} \mid \mathrm{L}_{1} \mathrm{~L}_{2}\right)$ and $p\left(\mathrm{R}_{2} \mid \mathbf{R}_{1} \mathrm{~L}_{2}\right)$. Of the ten pairs of subjects in this study there were eight pairs for which these propensities were defined for both players. In the other two pairs the outcome on which the propensity was conditional never occurred, hence the conditional probability was undefined. The product-moment correlation for those pairs in which both individual propensities were defined was $r=.96$ (significant beyond $p=.01$ ). Thus the answer appears to be straightforward; as Row 
player shows increasing readiness or likelihood of carrying out his threats, Column player shows an increasing likelihood of capitulating to Row once the threat has been carried out.

The high correlation between Row's readiness to carry out threats and Column's readiness to capitulate leaves unanswered the question of cause. Three alternative interpretations can be made.

1. A Row player who is likely to carry out threats causes Column to capitulate readily to these threats. This interpretation suggests that the "tougher" or more "reckless" Row appears, the more likely it becomes that Column will acquiesce by capitulation.

2. A Column player who readily capitulates reinforces Row's use of threat and thus encourages Row to make greater use of his threat in the future. The notion in this interpretation is that a "weak" Column player is one who not only gives in under threat but, by doing so, actually invites the Row player to carry out more threats.

3. Combining the previous two interpretations a third situation is seen in which Row's threats lead to capitulation by Column, and this capitulation in turn leads Row to carry out further threats. This interpretation takes the strategic interaction between Row and Column to be a cyclical one in which threats bring about concessions and these concessions in turn lead to further threats. Presumably, it is an interaction of this sort to which cautions against "rewarding aggression" are directed.

Since the correlation between threats and capitulation does not specify the direction of causation, any one of the above three interpretations may be correct. In order to decide which most accurately describes the interaction between Row and Column players, there is need for further experiments to be conducted in which the strategy choice behavior of one or the other player is under experimental control.

The strategic interaction that takes place in playing Game 21 centers around Row's ability to carry out threats against Column and Column's options of appeasing, capitulating, or resisting Row. One way to study this interaction is by looking at the matrix of transition probabilities from the outcome on trial $n-I$ to the outcome on trial $n$.

Table 3 shows the matrix of transition probabilities determined from the combined data of eight of the ten pairs of players each playing 300 trials of Game 21. Two pairs were excluded from this analysis because they showed no variation in their strategy choices, one pair always selecting the LL outcome and the other the LR outcome. In Table 3, each cell entry is the conditional probability of going to the column outcome on trial $n$, given that the row outcome occurred on trial $n-1$; from this data the following generalizations can be made: 
TABLE 3

Transition Probabilitieg from Outcomes on Trial $n-1$ to Outcomes on Trial $n$ for Eight of the Ten Pairs of Subjects

\begin{tabular}{llllll} 
& & \multicolumn{2}{c}{$n$} & \\
\cline { 2 - 3 }$n-1$ & $\mathrm{LL}$ & $\mathrm{LR}$ & $\mathrm{RL}$ & $\mathrm{RR}$ & $\mathrm{Pr}$ (outcome) \\
\hline $\mathrm{LL}$ & .77 & .11 & .09 & .03 & .65 \\
$\mathrm{LR}$ & .44 & .45 & .05 & .06 & .18 \\
$\mathrm{RL}$ & .43 & .14 & .33 & .10 & .12 \\
$\mathrm{RR}$ & .34 & .25 & .27 & .14 & .05 \\
\hline
\end{tabular}

1. Given that an LL outcome has occurred, Row is as likely to carry out his threat against Column on the next trial as Column is to appease Row on the next trial.

2. If Column has given Row his largest payoff on a trial, he is as likely to repeat this concession on the next trial as to withdraw it.

3. Given that Row has carried out his threat against Column, he is as likely to repeat it on the next as he is to withdraw it. (The probability of Row repeating his threat is equal to

$$
p(\mathrm{RL} \mid \mathrm{RL})+p(\mathrm{RR} \mid \mathrm{RL})=.43 .)
$$

4. From Table 3 it can be seen that there is a bias toward repetition of outcomes from the $n-1$ to the $n$th trial, indicated by the fact that each diagonal entry in the matrix of transition probabilities exceeds the corresponding row marginal probability. This repetition bias is weakest for the LL outcome; on the other hand, the probability of repetition of LR and RL is more than twice as great as would be expected if the probabilities of outcomes were independent of the prior outcome. Essentially what this says is that the occurrence both of threats by Row and concessions by Column tend to increase the probability of their subsequent recurrences. This provides some evidence that an aggiession-concession interaction between players tends to be self-reinforcing.

The results shown in Table 3 support the conclusions drawn earlier. Column playcr both appcases and capitulates; Row sometimes carrics out his threats against Column in an attempt to gain his largest payoff, and these attempts are often successful.

Our discussion of Game 21, both in terms of its strategic characteristics and of the behavior of naive subjects playing iterated trials of the game, brings up a problem alluded to earlier. This is the problem of what constitutes "rational" decision-making in a two-person nonzero-sum game.

A prominent feature of Game 21 is that each player has a dominating strategy choice, a choice which guarantees the player his larger payoff 
regardless of what the other player does. In a single play of the game, the logic of selecting the dominating strategy choice is compelling; regardless of what the other does, each is better off selecting the dominating rather than the dominated strategy. However, the compelling logic, as a method of formal analysis, can be extended to cover repeated plays of the game as well. The argument for this is identical to the one offered by Luce and Raiffa (1957, p. 98) in their discussion of repeated plays of the Prisoner's Dilemma game.

In Prisoner's Dilemma, as in Game 21, each player has a dominating strategy choice. In playing repeated trials of these games it can be argued that, regardless of what the players choose on the preceding trials, they each ought to select their dominating strategy choice on the final play. However, since the outcome of the final play is dictated by the rationality of selecting the dominating strategy, the players must consider what to do on the next-to-last play of the game. Here too, however, the logic of the dominating strategy is compelling since, regardless of what occurred on previous trials, one is always better off with the dominating strategy on trial $n-1$. Having covered trials $n$ and $n-1$, the argument moves to trial $n-2$, where the identical logic prevails. Ultimately, the consequence of this rational argument is that the dominating strategy choice should be selected by both players on each and every play of the game.

The naive subjects in this study who played repeated trials of Game 21 chose to defy the above logic. Both Row and Column departed quite liberally from the dominating strategies. These variations in play, while in contradiction to "rational" rules of decision-making, nevertheless appear to have a logic of their own, a psychological rather than a formal logic.

The psychological logic that justifies departures from the dominating strategy choices in Game 21 begins with the fact that Row is dissatisfied at the "natural outcome," i.e., the outcome that is determined by the intersection of the individual dominating strategy choices. Row has two reasons for switching from his dominating strategy. First, by doing so he reduces the difference between his own and the other's payoffs (this is not necessarily true of Game 21 per sc; it is truc for the particular assignment of interval scale payoffs assigned to Game 21 in this study). Since there is evidence that subjects playing nonzero-sum games evaluate their payoffs both in terms of absolute values and in terms of the difference between their own and the other's payoffs, Row gains some reward by reducing the difference between his own and the other's payoff. Second, and more important, Row, by switching from the natural outcome, indicates to Column that he will not "accept" the natural outcome. If he is able to convince Column of this, Column is faced with the choice of re- 
sisting Row and repeatedly receiving 6 points, or capitulating to Row, thereby receiving 15 points. What this comes to is that, if Row behaves "irrationally" and departs from his dominating strategy choice, and further, is able to convince Column that he will continue to act in such a manner, then it becomes reasonable for Column to depart from his dominating strategy choice, for by doing so he increases his own payoffs. A key factor in this type of interaction is that Row must make his threats credible; he must convince Column that he is prepared to act irrationally. Once convinced of this, a "reasonable" Column player will capitulate to Row and switch to his non-dominating strategy choice. Alternatively, a Column player faced with what appears to be an irrational opponent may choose to resist Row's threats. If Column makes no concessions to Row, he may succeed in convincing Row that he will never depart from his dominating strategy choice. If Row is so convinced, it then becomes Row's own interest to stop carrying out his threats and revert to his dominating strategy, thus gaining an increment in his payoff.

The departures by both Row and Column from their dominating strategy choices can be seen to be motivated by attempts to maximize individual payoffs. However, these attempts are represented by departures from, rather than conformity to, exclusive selection of dominating strategy choices. It is reasonable to expect that Row will act "irrationally" if Column fails to do so; it is reasonable for Column to act "irrationally" if Row begins to do so.

Because of the disagreement between normative decision-making criteria on the one hand, and "reasonable" departures from dominating strategy choices by either Row or Column on the other, Game 21 has the properties of a "dilemma."

In this study, nine of the ten pairs of subjects presented this dilemma "resolved" the dilemma by engaging in "reasonable" but "irrational" behavior. One pair of players behaved "rationally."

A final question remains: how, and to what extent, does the occurrence of "irrational" behavior affect the magnitude of payoffs achieved by the players?

In Figure 2, the total expected payoffs for each player's two pure strategies are plotted as a function of all possible probability mixtures of strategy choices of the other player. The expected values are based on the assumption that the probabilities in a strategy-choice mixture are independent and not contingent in any way on the other's choice.

From the figure it can be seen that Row's L strategy choice gives a larger expected value than his $\mathrm{R}$ strategy choice against any probability mixture that Column selects. This is equivalent to saying that strategy choice $\mathrm{L}$ dominates strategy choice $\mathrm{R}$ for Row. The situation is identical 


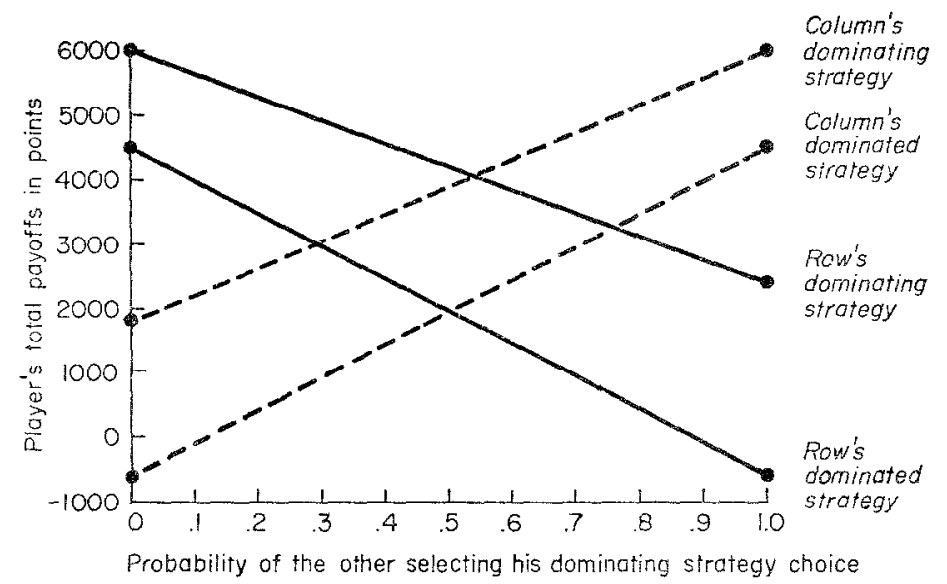

FIG. 2. The expected total payoffs for each of the two players' pure strategies as a function of all possible mixed strategies of the other player.

for Column; the curve of expected payoffs for his two strategies, plotted against all of Row's possible probability mixtures of strategy choices, also shows that $L$ gives a larger expected payoff than $R$ against any mixture that Row plays.

According to Figure 2, it is evident that if Column always selects his L strategy, Row can receive at most 2,400 points, and he can receive this much only if he too always selects the $\mathrm{L}$ strategy; if he departs from $\mathrm{L}$ and Column does not, his expected payoff will be reduced. Since $\mathrm{L}$ is a dominating strategy for Column, the normative solution of this game ought to be repeated LL outcomes, which gives Row 2,400 points and Column 6,000 points for the 300 trials of play. Against this normative solution, we compare the actual payoffs received by the ten pairs of subjects playing the game.

In Figure 3, the payoffs of the Row and Column players in each pair are shown. The pairs are ordered in this figure in terms of the magnitude of the Row player's payoff, i.e., the left-most pair (7) is the one whose Row player received the smallest payoffs of all Pow players; the rightmost pair (3) is the one whose Row player received the largest payoffs of all Row players. From Figure 3 it is seen that the normative solution was reached by only one pair of players (pair 4). Of the remaining pairs, there were six in which the Row player obtained payoffs greater than the maximum of 2,400 points dictated by the normative solution to the game. Now, Row can obtain payoffs in excess of 2,400 points only if Column. departs from his dominating strategy choice. On the basis of our previous analysis of Game 21, it was concluded that such departures by Column 


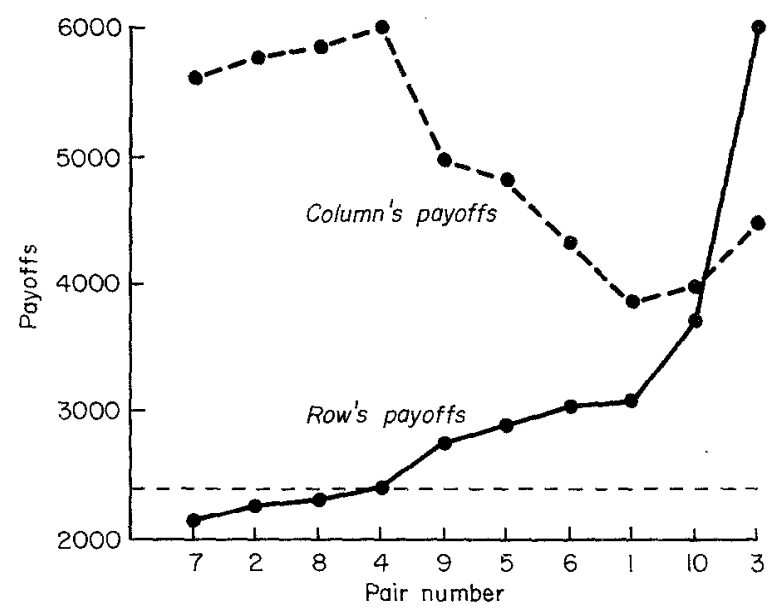

FIG. 3. The total abserved payoffs of Row and Column player in each pair for the 300 trials of play. (The horizontal dashed line indicates Row's maximum payoff if Column never departs from his dominating strategy choice.)

would occur as the result of either appeasement or capitulation. Thus, one way to determine the effectiveness of Row's threats as a means for increasing his payoffs is to examine the correlation between Row's likelihood of carrying out his threat, $p\left(R_{1} \mid L_{1} L_{2}\right)$, and the total payoff received by Row. The rank-order correlation between Row's likelihood of carrying out his threat and Row's total payoff across the eight pairs of players, for which $p\left(\mathbf{R}_{1} \mid L_{1} L_{2}\right)$ is defined, is $r_{\mathrm{s}}=.93$ (siguificant beyond $p=.01$ level). The implication of this high correlation is provocative in respect to the concept of rationality previously discussed. On the basis of the corrclation between $p\left(\boldsymbol{R}_{1} \mid \mathbf{L}_{1} \mathrm{~L}_{2}\right)$ and Row's total payoffs, it appears that Row's payoffs increase as his likelihood of departing from the "rational" strategy choice increases.

As mentioned previously, the game used in this study bcars certain similarities to the famous Prisoner's Dilemma game. In both games each player has a dominating strategy choice and each game represents a "mixed-motive" situation. The particular motives involved in each game differ but both pit a "rational" strategy against a strategy which offers the players an opportunity of earning more than they could earn by playing their rational strategies.

Studies of the behavior of naive subjects playing the Prisoner's Dilemma (see Rapoport \& Chammah, 1965) indicate that in that game, just as in Game 21, subjects, to a significant degree, do depart from the pattern of play prescribed by rational decision rules. In the Prisoner's Dilemma such departures represent "cooperative" behavior and may be 
adapted by the players when they realize that they both can earn more by jointly playing their non-dominating strategy choices rather than their dominating strategy choices. In Game 21, departures from the dominating strategy, though not cooperatively motivated, do have similar purpose, the increase of a player's earnings. And, as has been shown by the data presented in this study, s1ch departures from the rational strategy choice often are successful in giving Row player a payoff greater than he would receive if such departures were not made.

In general, caution must be exercised in attempting to generalize from the results of controlled laboratory studies of simple two-person games to more complex instances of social interaction. However, in the case of the present study, the simplicity of the game environment justifies and strengthens generalizations that may be made concerning the role that rationality plays as a determinant of mixed-motive behavior in real-world conflict situations. For the players of Game 21, the problem of strategy choice selection was simple and straightforward. The payoffs were clearly indicated; each player had to choose between only two alternatives, and the consequences of strategy choices were immediate and accurate. Yet it was evident that the simple principle of rationality that specified a normative outcome to this game proved wholly inadequate as a description of the strategy choice behavior of human players. It thus seems reasonable to conclude that, in the infinitely more complex arena of realworld conflicts, where payoffs, strategy alternatives, players, and states of information are constantly in flux, the "obvious" rationality of selecting dominating strategy choices would fail to stand out as an adequate description of human behavior.

\section{REFERENCES}

Gallo, P. S., JR., \& McClintock, C. G. Cooperative and competitive behavior in mixed-motive games. Journal of Conflict Resolution, 1965, 9, 68-78.

Iuce, R. D., \& RatFFA, H. Games and decisions. New York: Willey, 1957.

RApopont, A., \& CHAMmaH, A. 'The prisoner's dilemma: A study of conflict and cooperation. Ann Arbor, Mich.: University of Michigan Press, 1965.

RAPOPORT, A., \& GUYER, M. A taxonomy of $2 \times 2$ games. General Systems, 1966, 11, 203-214.

Rapoport, A., \& ORWANT, C. J. Experimental games: A review, Behavioral Science, $1962,7,1-37$.

(Received August 12, 1968) 